\title{
ALEXANDER VON HUMBOLDT Y AMADO BONPLAND: SU LEGADO EN LA REVALORACIÓN DE LA HISTORIA NATURAL
}

Alexander von Humboldt and Amado Bonpland: their legacy in the revaluation of natural history

\author{
RODRIGO CAJADE ${ }^{1}$ D \& ALEJANDRA B. HERNANDO ${ }^{1}$
}

\begin{abstract}
Resumen: La Historia Natural (HN) es una disciplina relevante de las Ciencias Naturales que se encarga de describir a los seres vivos y su ambiente a partir de la observación precisa. Como madre de las ciencias occidentales, sus orígenes se remontan a los escritos de Aristóteles, para atravesar desde entonces la historia de la humanidad brindando conocimientos esenciales para su desarrollo. Luego de su auge entre los siglos XVII y XIX con los viajes exploratorios de los naturalistas europeos a los nuevos continentes, la $\mathrm{HN}$ ha perdido valoración en el aporte a la construcción del conocimiento científico. Desde entonces el debate sobre el rol de la HN en las ciencias ha oscilado como un péndulo entre su estimación y desestimación. En esta contribución, presentamos los principales temas de este debate aportando nuestro punto de vista a partir de nuestra experiencia en la revaloración de la historia de la $\mathrm{HN}$, resignificada con la influencia de la obra de Alexander von Humboldt y Amado Bonpland. La especialización de las ciencias, los avances tecnológicos, la pérdida del sentido naturalista y la vinculación con el romanticismo han sido, entre otras, las principales causas que han desacreditado a la HN. Sin embargo, concluimos que la historia nos muestra que la $\mathrm{HN}$ ha sido y es fundamental para el crecimiento y desarrollo de la humanidad.
\end{abstract}

Palabras clave: Ciencia, descripción, educación, naturaleza, naturalistas.

\begin{abstract}
Summary: Natural History $(\mathrm{NH})$ is a relevant discipline of the Natural Sciences that describes organisms in their environment from accurate observation. As the mother of Western sciences, its origin dates back to Aristotle's writings. Since then it crossed human history providing essential knowledge for humanity development. After flourishing between the 17th and 19th centuries due to the exploratory travels of European naturalists to the new continents, $\mathrm{NH}$ has lost appreciation in its contribution to scientific knowledge. The debate about its role in sciences has swung like a pendulum between estimation and dismissal. In this contribution, we present the main themes of this debate. We expose our point of view from our experience that revalues the history of $\mathrm{NH}$ under the influence of Alexander von Humboldt and Amado Bonpland' works. The specialization of sciences, technological advances, the loss of the naturalistic sense, and the connection with romanticism have been among, the main causes that have discredited $\mathrm{NH}$. However, we conclude that history shows us that $\mathrm{NH}$ has been and is fundamental for the growth and development of humanity.
\end{abstract}

Key words: Description, education, naturalists, nature, Science.

${ }^{1}$ Laboratorio de Investigación en Diversidad, Ecología y Conservación de Vertebrados Alexander von Humboldt, Dep. de Biología, Facultad de Ciencias Exactas y Naturales y Agrimensura, CONICET, Universidad Nacional del Nordeste, Av. Libertad 5470, 3400, Corrientes, Argentina. E-mail: rodrigocajade@hotmail.com; abhernan@exa.unne. edu.ar 


\section{¿Qué es la historia natural?, ¿Qué hace un naturalista?}

"Todos al nacer somos historiadores naturales" (Anderson, 2013). Diferentes autores se han referido a la Historia Natural (HN) definiéndola de diversos modos (Schmidly, 2005; Anderson, 2013; Francioli, 2015). Ciertamente no existe una definición consensuada que satisfaga a todos (Tewksbury et al., 2014) y las definiciones varían según el objeto de estudio, la metodología y el campo de acción que la HN debería abarcar. Sin embargo, la mayoría de los autores coinciden en que la $\mathrm{HN}$ representa la práctica de la observación y descripción de la naturaleza, de los seres vivos y su entorno. Consecuentemente, quien observa y describe la naturaleza es un naturalista, es decir un practicante de la $\mathrm{HN}$. La conceptualización de la naturaleza es tan amplia y diversa que le otorga a la $\mathrm{HN}$ un carácter multidisciplinario y multiescala, atributo que refleja su gran alcance y potencial (Tewksbury et al., 2014). Pero este carácter diverso también evidencia la ambigüedad de su definición y representa un problema a resolver (Francioli, 2015). En muchos casos una carga subjetiva de valoración acompaña a las definiciones, sea para estimar o desestimar a la $\mathrm{HN}$ y al naturalista. Estas ambigüedades y subjetividades definitorias representan el eje central del debate sobre el rol que la $\mathrm{HN}$ y los naturalistas ocupan en las ciencias. De ese debate aquí nos ocuparemos.

La esencia de la HN está constituida por la observación libre de la naturaleza y por su principal fundamento metodológico, la descripción empírica, ambas con influencia directa en el origen y concepción de ideas para comprender el mundo natural. Ciertamente, esa libertad para la observación y descripción del mundo que nos rodea es una condición innata de la humanidad. Nacemos con la capacidad de observar siendo parte de la naturaleza, sin condiciones ni nociones preconcebidas para describir, narrar y transmitir nuestra experiencia. Tal como refiere Anderson (2013), "todos al nacer somos historiadores naturales", porque ciertamente todos al nacer somos naturalistas. Es por ello elocuente pensar que las ciencias hayan nacido con lo primero que el hombre siempre ha sabido hacer: observar, describir e interpretar para comprender el mundo natural $\mathrm{o}$, en otras palabras, practicar $\mathrm{HN}$.

A cada paso de la historia de la humanidad el amplio abanico de la HN se abre en nuevos conocimientos (Fleischner, 2001; Anderson, 2013). Desde las sociedades de cazadoresrecolectores, aquellas de la Antigua Grecia y del Imperio Romano, de la Europa medieval y de la Edad Moderna hasta la actualidad, la HN ha sido fundamental para el desarrollo del ser humano. En los inicios de la humanidad, para una tribu de cazadores-recolectores tener un conocimiento amplio de un conjunto de especies animales y vegetales así como de la obtención de agua y refugios, entre otros recursos, implicaba la esencia misma de su supervivencia (Fleischner, 2005; Anderson, 2013). Observar y describir para sobrevivir era una cuestión fáctica que demuestra el poder irrefutable de la HN (Fleischner, 2005).

En los anales de las ciencias, la HN representa la piedra fundacional de las Ciencias Naturales occidentales, la más antigua y madre de todas las ciencias (Fleischner, 2005). Su práctica científica se remonta a las escrituras de Aristóteles (384-322 a. C.), quien abandonó la justificación de los fenómenos a través de las creencias para dar paso a la explicación racional por medio de la evidencia que brinda la práctica de la observación y experimentación. Aristóteles se ocupó, entre otras cosas, de describir los organismos y agruparlos según sus atributos dando origen a las primeras clasificaciones de los seres vivos. Su legado influiría a sus sucedáneos inmediatos y a otros más allá de los siglos. Pero, la $\mathrm{HN}$ recibiría su bautismo formal como rama de las ciencias con el enciclopédico trabajo de 37 tomos del romano Plinio el Viejo (23-79 d. C.). Con su obra Naturalis Historiae, se acuña el término por primera vez para describir la naturaleza tal como era concebida en esa época (Anderson, 2013).

Su auge entre el siglo XVII al XIX promovió su diversificación y fue el origen primordial de la Anatomía, Taxonomía, Zoología, Botánica y Geología. A partir de la revolución lineanna (siglo XVIII) con las ciencias de la clasificación y de los viajes exploratorios a los nuevos continentes, la $\mathrm{HN}$ corrió el velo enceguecedor 
del credo cristiano y reveló ante los propios entusiastas y escépticos las maravillas del mundo natural, "había todo un universo de especies animales, vegetales, de territorios, de naturaleza salvaje, más allá de lo que los sagrados testamentos profesaban" (Anderson, 2013). Este tiempo prolífero de la HN ocurrió durante el surgimiento de la Ilustración o Período de las Luces, durante el cual el hombre incrementó su saber en grandes proporciones luego de haber atravesado la oscura Edad Medieval, sin grandes descubrimientos para la ciencia y signada por la cosmología religiosa y supersticiosa de los fenómenos naturales. A partir del siglo XVIII, la HN amplió los conocimientos del hombre dándole un orden basado en las clasificaciones y colecciones fomentadas por los descubrimientos en el Nuevo Mundo, un nuevo conocimiento que brindó a los europeos una gran variedad de alimentos, minerales y medicinas, hasta entonces desconocidos, provenientes de la naturaleza descubierta por los naturalistas. A partir del siglo XX comenzaría un período que se extiende hasta la actualidad en donde la $\mathrm{HN}$ sería cuestionada como disciplina científica y perdería créditos sobre los nuevos avances y logros del desarrollo humano (Schmidly, 2005; Francioli, 2015).

La historia de la HN (Anderson, 2013) revela su perfil dinámico influenciado por los diferentes contextos históricos geopolíticos, las corrientes filosóficas culturales y la religión, adaptándose a cada tiempo, mientras que nuestra historia como especie, nos enseña que el incremento de nuestra supervivencia ha dependido, en gran parte y en todo momento, del conocimiento que brinda la HN (Tewksbury et al., 2014). Desde nuestros inicios como especie humana o desde el momento en que alguien nace, el naturalista y la $\mathrm{HN}$, tan antiguos como actuales, representan en sí mismos una expresión de la esencia humana.

\section{La historia natural ¿una ciencia desacreditada? El debate}

"La ciencia es como una pirámide, mientras muchos científicos se hallan enamorados en experimentos y teorías en la cima de la pirámide, toda la empresa se derrumba sin la base sólida de la descripción y comparación" (Fleischner, 2005). "Detrás de una teoría hay evidencia que la historia natural proporciona y la sostiene" (Fleischner, 2005). A pesar de la contundente veracidad de esta frase y de las distintas iniciativas que han expuesto el valor de la $\mathrm{HN}$ para las ciencias, defendiendo su práctica y promoción (Tewksbury et al., 2014), el valor de la $\mathrm{HN}$ es continuamente cuestionado en el ámbito científico-académico. Desde la década de 1990 la HN fue objeto de un intenso debate sobre su extinción como disciplina científica, debate que como un péndulo oscila entre su estimación y su desacreditación (Noss, 1996; Futuyma, 1998; Arnold, 2003; Fleischner, 2001; Greene, 2005; Schmidly, 2005; Francioli, 2015; Barrows et al., 2016). El debate ha sido consecuencia del propio avance en la teorización de las ciencias (ej. introducción de la Matemáticas en la Biología, hecho que separó a la Biología de las Ciencias Físicas), el avance técnico asociado a las metodologías de estudio (ej. surgimiento de la Biología Molecular), la diversificación e hiper-especialización disciplinar (aumento de la masa crítica de conocimiento e investigadores), aspectos históricos socioeconómicos (despoblamiento de los campos hacia las ciudades) e históricos geopolíticos (la segunda guerra mundial y la guerra fría con la necesidad de una ciencia con resultados en la inmediatez). Con el avance en la Matemática el refinamiento teórico de las ciencias introdujo la complejización de los estudios cuantitativos y la experimentación, resultando en un tipo de ciencia especializada o ciencia dura (Schmidly, 2005). La tecnofilia, tal como refieren algunos autores, y la hiper-especialización del siglo $\mathrm{XX}$ condujo a una diversificación de ramas científicas tan refinadas que resulta difícil establecer su conexión con las raíces de origen (Francioli, 2015). Al respecto, es interesante notar que el número de ramas científicas pareciera incrementarse con cada uno de los nuevos avances tecnológicos emergentes o con nuevas aplicaciones metodológicas. Este hecho puede fácilmente observarse con el surgimiento de revistas científicas ultra especializadas asociadas a una nueva tecnología. Un ejemplo elocuente y actual, entre tantos, es la revista científica Environmental DNA que publica 
desde el año 2019 artículos exclusivos que aplican la metodología de ADN ambiental. La pregunta es, ¿por qué se editan este tipo de revistas ultra-especializadas si los trabajos en ellas publicados bien pueden encuadrar en muchas otras de prestigio, alcance general y competencia disciplinar? La sensación de esta coyuntura actual de las ciencias, es que la conjunción de nuevas tecnologías y/o metodologías en la creación de nuevas ramas científicas despojan al científico de la visión panorámica indispensable para la comprensión biológica al tiempo que éste se ve info-abrumado por la diversidad de especializaciones.

En el ámbito de las grandes revoluciones de la humanidad (agrícola, industrial y tecnológica) la $\mathrm{HN}$ fue perdiendo terreno en su ponderación para los seres humanos. El surgimiento de la agricultura que permitió el acopio de alimento y el establecimiento fijo en asentamientos permanentes donde cultivar comenzó a diluir la importancia que la $\mathrm{HN}$ tenía para las sociedades de cazadores-recolectores. La atención se focalizó en un pequeño conjunto de especies que proveían semillas para los cultivos (Anderson, 2013). Así, comenzó el camino hacia el sedentarismo y la noción del ser humano como parte de la naturaleza cambió radicalmente para iniciar con su dominio. Con la revolución industrial, la concentración de las grandes fábricas en los centros urbanos y la promesa de trabajo produjeron grandes migraciones hacia las ciudades. La maquinaria industrial potenció la agricultura al reducir tiempo y costos promoviendo una industria de alimentos que puso al alcance de la mano la obtención de aquellos antes producidos a pequeña escala en los hogares u obtenidos de la naturaleza. Como consecuencia, la desvinculación física y emocional de la naturaleza inició un proceso de divorcio que despojó al hombre del sentido de su observación, apreciación y valoración. Esta disociación con la naturaleza no solo sustrajo al hombre de su sentido naturalista, reduciendo el número de potenciales practicantes de $\mathrm{HN}$, sino que además es la responsable de las mayores atrocidades cometidas por el hombre contra la naturaleza. El hombre promedio del siglo XXI se ha vuelto ajeno a ella y su estimación se redujo a valorarla desde un punto de vista recreativo. Es interesante ver cómo interactúan las diferentes causas que han incidido sobre la valoración de la HN. La pérdida del contacto con la naturaleza también es promovida en el ámbito científico por la tecnofilia y la sobrestimación de la modelización de datos con la consecuente reducción de trabajo de campo, que conduce ineludiblemente a la pérdida de una correcta interpretación de la naturaleza (Noss, 1996; Futuyma, 1998). Por ejemplo, es habitual observar en trabajos sobre modelos de distribución geográfica, hábitats $\mathrm{y}$ ambientes, entre otros, que los datos y proyecciones realizados no han sido cotejados por observaciones de campo. Entonces ¿cuál es el sentido de proyectar la distribución geográfica de una especie sobre la base de las condiciones ambientales utilizadas por el modelo si éstas no se corresponden con la realidad, en la naturaleza? ¿Cuál es el sentido de describir el uso de hábitats y planificar estrategias de conservación de una especie a partir del modelado, si no se han cotejado en el campo datos de la especie en asociación con los hábitats? Aunque parezca asombroso, numerosos trabajos sin cumplir estas premisas fundamentales para la generación de un conocimiento real, son publicados cada año en revistas muy ¿prestigiosas?, según los índices de rankings conocidos.

Otro argumento del debate para desacreditar la $\mathrm{HN}$ es su vínculo con las subjetividades propias de la expresión de las sensibilidades. Durante su auge, en la transición de la Ilustración al Romanticismo, los naturalistas con sus viajes exploratorios tiñeron a la $\mathrm{HN}$ con una nueva manera de ver el mundo: la adición de un componente expresivo, propio a la sensibilidad del observador naturalista, a su valorada descripción racional de alta precisión. Adicionalmente, la aventura romántica de los viajes exploratorios y la apertura de las ciencias a la sociedad condujo a un incremento en el interés por la descripción de la naturaleza, volviendo a la $\mathrm{HN}$ como la ciencia ideal para ser practicada desde el amateurismo por aficionados. El posterior surgimiento de la ciencia física moderna con la profundización de la matemática exigió a la $\mathrm{HN}$ escapar del romanticismo como si fuera un mal sueño (Dettelbach, 1999). Sin embargo, ¿desafortunadamente?, su vinculación con el 
romanticismo ha permanecido en el tiempo como un fantasma, dificultando su aceptación como disciplina científica entre los académicos. Como consecuencia del romanticismo y la popularización de las ciencias, se construyó una idea menospreciada de la HN calificándosela como anticuada y anecdótica. En estos términos, la conceptualización del naturalista ha adquirido una mirada peyorativa, o en el mejor de los casos, pintoresca, romántica "propia de los amantes de la naturaleza" (Schmidly, 2005). Injustamente se ha considerado a la práctica de la $\mathrm{HN}$ tan subjetiva a la expresión de las sensibilidades como consecuentemente a causa de ello, objetiva a la falta de rigor científico e intelectualidad.

En nuestra apreciación, la $\mathrm{HN}$, como un ser vivo nació, creció, floreció y brindó sus frutos. Sin embargo, aunque pareciera hoy marchitarse, sus semillas sembradas por las obras de los naturalistas a través de la historia de la humanidad aún germinan en las ciencias actuales gracias al esfuerzo de quienes reivindican su práctica científica y resignifican su valor en la historia de las ciencias. El mundo continúa siendo terreno fértil para la $\mathrm{HN}$ y los beneficios de la observación precisa de los organismos en su ambiente continúan siendo prolíferos para la salud humana, seguridad alimentaria y conservación de la naturaleza (Tewksbury et al., 2014). Con otros matices, relevancia y adaptada a las tecnologías actuales, la HN continúa su curso en la construcción del conocimiento.

\section{El poder del naturalista y la historia natural}

La tarea del naturalista es la de ver lo "invisible" (Fleischner, 2001). El poder de la $\mathrm{HN}$ es inmutable, su carácter vital tan simple como contundente reside en constituir la esencia, la forma más elemental para la creación y desarrollo del conocimiento. Desde la década del '90, más de 400 trabajos desencadenaron el debate sobre su valoración en el marco de las ciencias (Francioli, 2015) el cual se generó por cambios ajenos a sus cualidades, a su esencia. La HN siempre estuvo allí, está y siempre estará presente, aún cuando surjan nuevas teorías, metodologías y tecnologías que refinen a las ciencias. El debate resurgirá por estos nuevos acontecimientos, no por una modificación de su esencia. Más allá de la importancia en su cualidad de descripción precisa, de su legado en los registros históricos y geográficos, de sus colecciones biológicas, la $\mathrm{HN}$ posee en su práctica un valor intrínseco que reviste al naturalista de cualidades únicas.

¿Qué distingue a un naturalista científico de un científico que no es naturalista? Es el poder de ver lo invisible porque es dueño de su experiencia observacional y de la creación del conocimiento, no de un conocimiento adquirido a través de la experiencia ajena. Ver lo invisible es ver más allá de la experiencia observada, se trata de lo que vio Arquímedes al observar la bañera, Newton al observar el manzano, Humboldt al observar el Chimborazo, Darwin al observar los pinzones de las Galápagos, o Einstein el reloj de Berna. Tal como refiriera Humboldt, "con el conocimiento llega el pensamiento, y con el pensamiento, el poder" (Wulf, 2016). Dueño del conocimiento, es quien lo construye por su cuenta, quien observa produce conocimiento y se impregna de poder. Por ello, es innegable el valor de la propia experiencia porque confiere al naturalista de una capacidad interdisciplinaria y de la facultad de una visión global e integrativa de la naturaleza. La observación de un gran abanico de fenómenos individuales comprendidos en la totalidad del conjunto, le permite realizar una interpretación conceptual de la naturaleza (Greene, 2005). Hayes (2009) denominó a esta capacidad de construcción intelectual interdisciplinaria como "inteligencia naturalista". Un naturalista observa con la razón pero también nutre su observación con las sensaciones y es parte de su capacidad el vínculo emocional proporcionado por la experiencia (Greene, 2005). En ese proceso de observación personal es donde los conocimientos preexistentes convergen, interactúan y promueven el desarrollo de nuevas ideas que conducen a una visión enriquecida que trasciende a la observación racional per $s e$, y producen una conceptualización integral del mundo natural. ¿Es entonces la crítica a la expresión subjetiva de las sensibilidades en realidad una virtud? ¿Hasta qué punto la subjetividad y la sensibilidad del observador pueden aceptarse en un naturalista científico? ¿Qué rol puede estar ocupando la subjetividad 
de la experiencia en el proceso de construcción científica del conocimiento? ¿Es posible que las condiciones ambientales durante el proceso de la observación de la naturaleza incidan en el desarrollo de procesos cognitivos que conduzcan a la construcción de pensamientos e ideas para la descripción del mundo natural y su interpretación de un modo diferencial naturalista? Cómo, cuáles, cuándo y qué procesos ocurren en la psiquis del observador durante su contacto con la naturaleza para incidir en la construcción de sus ideas y conocimientos es una tarea pendiente que sin duda contribuirá con la valoración del naturalista científico. Tal como plantea Francioli (2015), no solo desde las ciencias biológicas sino a partir de la intervención de otras disciplinas científicas podremos responder a la pregunta ¿qué es ser un naturalista?

\section{El problema de la enseñanza en las universidades}

Los naturalistas están muriendo y tienen pocos herederos. ¿Será la próxima generación de biólogos conservacionistas nada más que un grupo de nerds informáticos sin conocimiento de primera mano de la historia natural? ¿Se deduce que, por lo tanto, no tendrán vínculos emocionales personales con la tierra? (Noss, 1996).

El debate sobre la HN y su rol en las ciencias actuales incluye su enseñanza en la escuela y en la educación superior. Según Francioli (2015) existe una relación articulada entre la idea de la HN como resabio disciplinar conceptualmente ambiguo y la falta de su enseñanza en las universidades. Concretamente, una acusada ausencia de su competencia disciplinar ha causado la reducción o anulación de cursos y actividades de campo para los alumnos, arrastrando a sus disciplinas más afines como la Zoología, la Botánica, Geología, Taxonomía y Sistemática, entre otras. Al reducirse esta fuente de aprendizaje ¿cuál es la consecuencia para los estudiantes? Muchos autores sostienen que la disminución en el ámbito académico del trabajo de campo, territorio del naturalista, tendrá consecuencias negativas en la educación $\mathrm{y}$ formación de las nuevas generaciones de investigadores (Noss, 1996; Futuyma, 1998;
Anderson, 2017). ¿Quién puede negar el valor del trabajo de campo, las visitas a áreas naturales protegidas o a museos de historia natural? La profunda huella cognitiva y emocional de tales experiencias fue claramente ejemplificada por Anderson (2017) "quien haya visto la exaltación de un estudiante anillando por primera vez un ave durante un trabajo de campo comprenderá el impacto positivo de esa experiencia en sus actitudes posteriores hacia la naturaleza y la práctica de la ciencia". De persistir la deficiencia de la enseñanza de la $\mathrm{HN}$ en las universidades continuará latente la amenaza sobre su subsistencia en el ámbito académico. El riesgo de que las próximas generaciones de profesores universitarios se compongan de científicos y docentes que no han tenido entrenamiento adecuado en la teoría y práctica de la HN durante su formación desestimaría en ellos la enseñanza en sus alumnos y, así, una retroalimentación negativa llevaría a la total pérdida de la formación de científicos naturalistas (Anderson, 2013). Pero, ¿estos temores están empíricamente fundados o es una conjetura de la percepción de un problema? La realidad muestra escasos trabajos que fundamenten con datos la situación del problema (Francioli, 2015). En una investigación basada en encuestas a científicos y profesionales de las Ciencias Ambientales en California, el 93\% de los encuestados $(n=185)$ sostuvo que la $\mathrm{HN}$ es relevante para la ciencia y esencial o deseable en su formación. Sin embargo, más de la mitad se sintió inadecuadamente entrenado para impartir su enseñanza. Los resultados exhiben una notoria desconexión entre la percepción del valor de la $\mathrm{HN}$ y la oportunidad de adquirir su entrenamiento a través de la educación (Barrows et al., 2016). Las conclusiones de este estudio deben motivarnos a abordar el problema seriamente teniendo como eje fundamental para el trabajo a futuro el análisis de datos concretos para conocer con precisión la realidad en cada país, región y universidades. Estamos aún a tiempo.

\section{Resignificar la historia de la historia natural, nuestra experiencia}

"Salimos de una cadena infinitamente larga de eventos y personas, y también somos 
eslabones de una cadena que se extiende hacia un futuro infinito..... Puedo contarles historias a mis alumnos, y tal vez algún día mis alumnos contarán a otros historias de historias de historias. La cadena continúa mientras mantengamos los enlaces" (Anderson, 2013). La influencia de las obras de $\mathrm{HN}$ data desde sus propios orígenes. Como una cadena de conocimiento, los primeros eslabones entrelazados fueron las obras de los fundadores de la $\mathrm{HN}$ que promovieron y motivaron aquellas de sus sucedáneos hasta alcanzar una diversificación de influencias que se expande y transcurre hoy en un amplio tejido de conocimientos (Anderson, 2013). Sus agujas tejedoras son aún hoy la admiración y el entusiasmo por dichas obras. Así, el valor de la HN se resignifica y su valoración actual extiende su influencia como un legado.

A partir del descubrimiento biológico del ecosistema de cerros aislados del Paraje Tres Cerros (Corrientes, Argentina) que en 2010 se inició con la descripción de la fauna de anfibios y reptiles, nuestro tejido de conocimiento se unió a la obra de Humboldt y Bonpland. Motivados por la ausencia de antecedentes sobre estudios zoológicos en el área, comenzamos a indagar en la historia científica de este sitio maravilloso de nuestro litoral argentino. En ese recorrido hallamos que los únicos antecedentes de investigación científica habían sido realizados por botánicos. La descripción de tres especies endémicas nos llenó de expectativas para la propia fauna. Pero, particularmente, la referencia histórica de un botánico nos llamó la atención y sería determinante para el futuro de nuestro proyecto científico. Durante sus años viviendo en Santa Ana (hoy localidad denominada Bonpland, provincia de Corrientes), Amado Bonpland visitó el Paraje Tres Cerros. Sus referencias hacia la fauna, flora y potencial mineralogía despertaron nuestra curiosidad por indagar sobre la vida y obra del sabio francés y nos condujo ineludiblemente al siguiente eslabón en la cadena de conocimientos de la $\mathrm{HN}$ : Alexander von Humboldt.

Alexander von Humboldt y Amado Bonpland fueron artífices del arquetipo del viaje naturalista de los siglos XVIII y XIX (Penchaszadeh et de Asúa, 2010). Como hijos románticos de la ilustración, ambos naturalistas reflejan precisamente el sentido dual con el que hasta hoy se caracteriza al quehacer de un naturalista: la experiencia de una mirada sensible de la naturaleza acompañada de la medición reiterada y precisa. Encontramos en ellos la esencia del debate sobre la práctica de la $\mathrm{HN}$ y el naturalista. Alexander von Humboldt, es el hombre centro del debate. Los intentos por despojar a la $\mathrm{HN}$ del romanticismo fueron incluso abordados por los creadores de la ciencia humboldtiana, quienes llevaron a cabo sus esfuerzos en pos de librar a Humboldt de sus "sensibilidades científicas" (Detembach, 1999). Pero, respaldada con sus exhaustivas mediciones de múltiples variables para describir el mundo natural ¿quién podrá negar la ciencia de Humboldt por tener una percepción tan sensible que dotó a su pluma de una magia seductora para legarnos las obras más atractivas de HN? En ese intento de revisionismo histórico la intencionalidad de despojar el costado romántico en la deconstrucción de Alexander von Humboldt es una paradoja (Fig. 1). Su pluma y su palabra expresiva destacaron ¡tantas veces! el valor de las emociones de la experiencia en la construcción del conocimiento...

Desde el momento en que descubrimos la filosofía científica de Humboldt y Bonpland, la admiración por sus obras generó un entusiasmo que impactó en nuestros pensamientos e ideas. La visión de Humboldt y Bonpland de un mundo natural interconectado y propio al espíritu del hombre nos guiaría a continuar con un ambicioso proyecto de describir la flora y la fauna del Paraje Tres Cerros, sus interacciones con el medio y la sociedad. Este ecosistema que había sido (casi) ajeno a la lupa de las Ciencias Naturales, era una oportunidad única e inmejorable para ser estudiado con todo el poder de la HN. Observación y descripción precisa para poder comprender su mundo natural, darlo a conocer para generar en la comunidad local y regional un sentido de pertenencia y amor por la naturaleza, con la finalidad de promover su valoración y conservación fueron los objetivos trazados. Desde entonces, la articulación interdisciplinaria e interinstitucional permitió abordar el estudio de los diferentes grupos de 


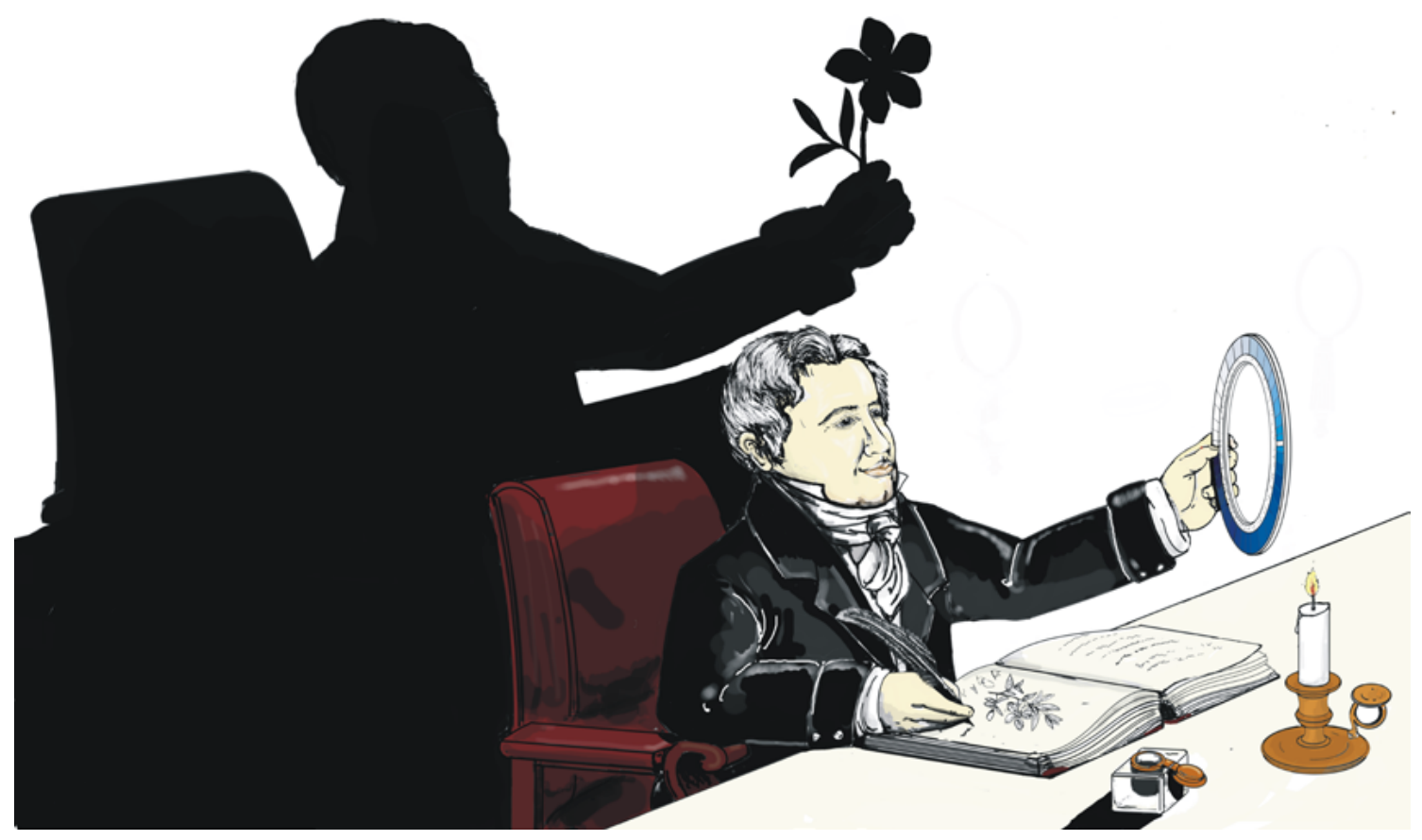

Fig. 1. El espíritu romántico de la ciencia ilustre de Humboldt se retrata en esta imagen. Mientras observa el valor del azul del cielo en el cianómetro, la expresión de sus sentimientos al rememorar la naturaleza se proyecta en su sombra como una flor, símbolo de la belleza. Paradójicamente, la dualidad de la precisión-expresión de Humboldt se resume en el cianómetro, instrumento de medición ya no utilizado en la actualidad por ser su medición del azul del cielo subjetiva a la mirada del observador.

Fig. 1. The romantic spirit of Humboldt's illustrious science is portrayed in this image. As he observes the value of the blue of the sky in the cyanometer, the expression of his feelings in recalling nature is projected in its shadow like a flower, symbol of beauty. Paradoxically, the duality of Humboldt's precision-expression is summarized in the cyanometer, a measuring instrument no longer used today because its measurement of the blue of the sky is subjective to the gaze of the observer.

plantas y animales desde diversas perspectivas. En ese camino recorrido, asumimos el compromiso de involucrar a los estudiantes de Biología a través de prácticas en trabajos de campo y de cursos sobre gestión de la naturaleza. A partir de estas experiencias varios de ellos fueron impregnados por el poder de la $\mathrm{HN}$ y posteriormente realizaron sus trabajos finales de graduación o doctorado abordando temáticas de la fauna o flora de los afloramientos rocosos del Paraje Tres Cerros. Por otra parte, el desarrollo del proyecto condujo a los investigadores y docentes a un proceso reflexivo sobre el alcance directo de nuestras investigaciones en la valoración y conservación de su singular naturaleza y cuáles serían los límites de nuestro compromiso para lograrlas. Coincidimos unánimemente junto con otros 11 colegas de la comunidad científica de Corrientes en que para canalizarlo debíamos contar con una herramienta de gestión que profundizara dichos alcances. Así, se formalizó la organización de la Fundación Amado Bonpland (FAB), una ONG sin fines de lucro dedicada a la investigación y conservación de la naturaleza. El intercambio y difusión de conocimientos con la comunidad local promovió un sentido de pertenencia destacado, reflejado en acciones concretas de algunos propietarios de las tierras tal como la creación, asistida por la FAB, de una reserva privada: la "Reserva Natural Privada Paraje Tres Cerros". Durante todo el proceso de investigación, extensión y transferencia, Humboldt y Bonpland estuvieron siempre presentes guiando nuestras 
ideas desde las suyas. Trabajando en este ecosistema de elevaciones rocosas, estuvimos permanentemente cautivados por el concepto de "Naturgemälde" (Cuadro de la Naturaleza) acuñado por Humboldt en su obra Ensayo sobre la geografía de las plantas. Tal como refiere Wulf (2016) en su biografía, en pleno auge de las clasificaciones taxonómicas con listas interminables de especies, Humboldt se interesaba por develar las relaciones entre las especies con el mundo natural. En el momento en que los científicos competían por realizar la lista más larga de especies, la genialidad de Humboldt para su época fue describir su interpretación del mundo natural... ¡con un dibujo! La idea del "Naturgemälde" fue concebida por Humboldt en el Chimborazo, fuerza natural invisible que canalizaría su genialidad creadora (Fig. 2). Su innovación gráfica representaría la muestra más cabal de su ciencia: la expresión del impulso de convertir las mediciones precisas en escritura, hacer que la naturaleza se escriba y dibuje, para que la variación de las fuerzas físicas "se manifestara a los sentidos" (Dettelbach, 1999).

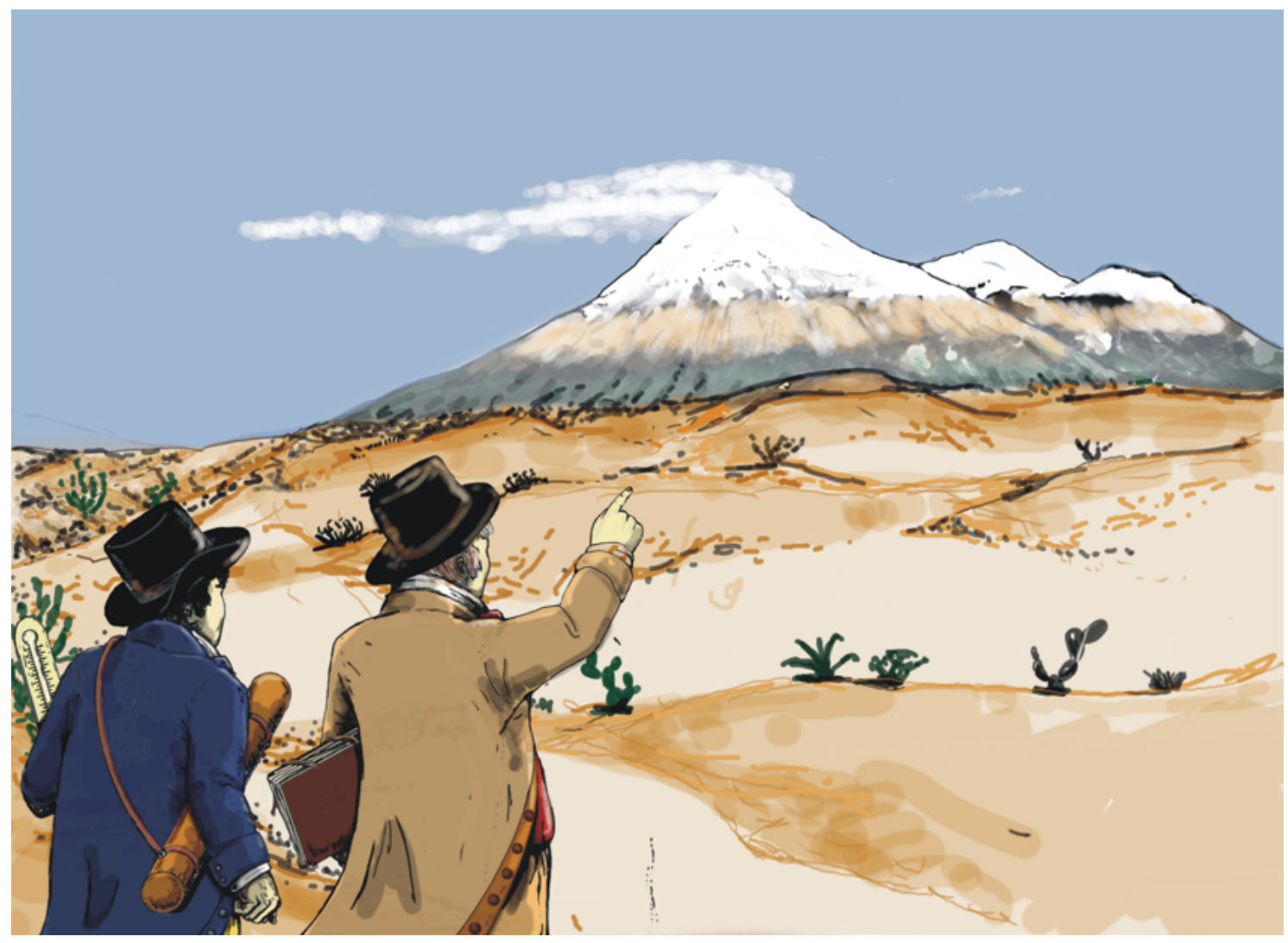

Fig. 2. Humboldt y Bonpland se aproximan al imponente Chimborazo en Ecuador. La visión panorámica desde la inmensidad de las alturas de este volcán evocaría el sentimiento de "Fernweh" en Alexander von Humboldt. Esas ansias y atracción por lo desconocido que había acumulado durante años de su juventud convergerían en una fuerza inspiradora al observar la infinitud del horizonte desde el punto más alto al que ningún ser humano había llegado antes. Fig. 2. Humboldt and Bonpland approach the imposing Chimborazo in Ecuador. The panoramic view from the immensity of the heights of this volcano would evoke the feeling of "Fernweh" in Alexander von Humboldt. Those longings and attraction for the unknown that he had accumulated during years of his youth would converge into an inspiring force as he observed the infinity of the horizon from the highest point that no human being had ever reached before. 
Humboldt y Bonpland no quedaron relegados únicamente al plano de las influencias científicas. A menudo nos conducían a la imaginación de situaciones históricas hipotéticas. ¿Habría mencionado Bonpland en sus cartas a Humboldt sobre este lugar tan fascinante de nuestro litoral? ¿Habría realizado Humboldt alguna apreciación al respecto? ¿Qué hubiese sucedido si Humboldt viajara a la Argentina para conocer $\mathrm{y}$ describir junto con su amigo Bonpland la naturaleza de cada uno de los tres cerros aislados del Paraje Tres Cerros? Nuestra respuesta fue unánime: probablemente hubiesen realizado un "Naturgemälde" de los tres cerros (Fig. 3).

El poder de las influencias de estos naturalistas nos brindó el entusiasmo y la determinación necesaria para dar un espacio al debate de la $\mathrm{HN}$ en nuestro ámbito académico. Juzgamos que el relato de la historia de la $\mathrm{HN}$ debía ser el primer paso para motivar a nuestros estudiantes en su práctica. Así fue que en 2017 un conjunto de profesores-investigadores de la FaCENA-UNNE, miembros de la Fundación Amado Bonpland y de la Asociación Cultural Bonpland organizamos un ciclo de conferencias con periodicidad mensual para resignificar el valor de los grandes naturalistas de fin del siglo XVIII y siglo XIX que realizaron importantes contribuciones en América Latina. Nuestro fundamento ante las autoridades académicas como respuesta a ¿por qué hacer un ciclo de conferencias referente a los grandes naturalistas que descubrieron la naturaleza de América Latina? fue:

"Como en cualquier proceso de desarrollo general del conocimiento gestado a lo largo del tiempo, conocer la historia de los pioneros que descubrieron el mundo natural de nuestro continente nos permite abordar una compresión más amplia de nuestro conocimiento actual sobre su naturaleza. Conocer esta historia no solo es un momento de aprendizaje sino que también es un acto moral que nos permite valorar, referenciar, resignificar y reivindicar el presente de nuestra profesión como biólogos. Aún hoy entre los estudiantes de Biología, es poco conocida la historia de los grandes naturalistas que con sus estudios contribuyeron al conocimiento de la naturaleza de la región. Desandar el recorrido de estos pioneros que transitaron por América Latina es también una invitación a conocer a la historia natural como disciplina madre de las Ciencias Naturales y una manera de visualizar sus alcances y su rol como sustento de las ciencias explicativas y aplicadas. ¿Por qué solo de América Latina? Porque fue y sigue siendo el jardín del planeta tierra, donde los grandes pensadores de la

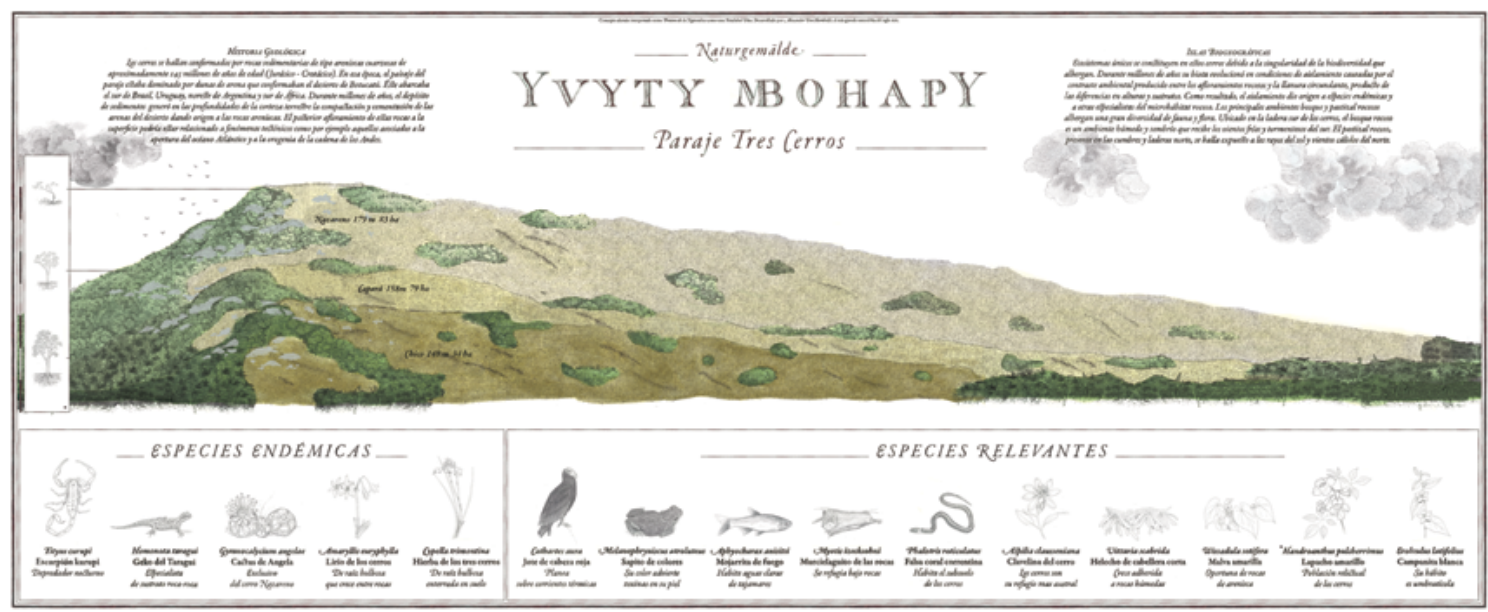

Fig. 3. "Naturgemälde" del Cerro Nazareno, Cerro Capará y Cerro Chico (Paraje Tres Cerros, Corrientes, Argentina, extraído de Cajade et al. (2019).

Fig. 3. "Naturgemälde" of Cerro Nazareno, Cerro Capará and Cerro Chico (Paraje Tres Cerros, Corrientes, Argentina, extracted from Cajade et al. (2019). 
historia natural encontraron el contexto de una naturaleza majestuosa, fuente de inspiración para consagrar sus ideas, sus acciones $y$ su legado. Por ello, esta propuesta propone un recorrido histórico sobre las obras de naturalistas y exploradores de los siglos XVIII y XIX que se destacaron por su trabajo en América del Sur tales como Amado Bonpland, Alexander von Humboldt, Charles Darwin, Alcides D'Orbigny, Félix de Azara y Florentino Ameghino".

El ciclo de conferencias fue un éxito rotundo. La audiencia se repartió entre estudiantes, profesores $\mathrm{y}$, felizmente, ciudadanos ajenos a la academia. La semilla de la HN comenzaba a germinar.

\section{El resplandor del conocimiento: cosechando el legado de Humboldt y Bonpland}

"La naturaleza, en todas partes, se dirige al hombre con una voz que es familiar para su espiritu" (Humboldt, 1814-1829). Con este breve resumen de nuestra experiencia, es elocuente cómo resignificar la historia de Humboldt y Bonpland con la práctica de la $\mathrm{HN}$ se promovió el descubrimiento del fascinante mundo natural del Paraje Tres Cerros y, sin dudas, un aporte en la construcción e incremento del conocimiento. Continuamente nos vimos embebidos en la fascinación de la observación libre y el descubrimiento de la naturaleza, bajo los ideales y concepciones de la práctica científica profesada por Humboldt y Bonpland. De estos hijos románticos de la ilustración, aprendimos su precisión, tenacidad y perseverancia. El esfuerzo del trabajo de campo para el registro de incontables variables nos permitió publicar trabajos científicos de gran valor, pioneros para el Paraje Tres Cerros. Pero, también aprendimos de la delicada pluma de Humboldt para contagiar sensibilidad durante la extensión de los resultados y que éstos sean rápidamente absorbidos por la comunidad en el proceso de valoración del Paraje Tres Cerros.

El debate es una condición sine qua non del enriquecimiento en la práctica y teorización de las ciencias. Seguramente, el péndulo de la valoración de la HN seguirá su curso con el tiempo. Sin embargo, creemos que un debate carece de sentido cuando quienes participan de él no intentan empatizar con las posturas opuestas. Quienes somos partidarios de la valoración de la $\mathrm{HN}$, debemos reconocer que tanto ésta como las ciencias experimentales son parte importante de un mismo proceso de desarrollo del conocimiento y por lo tanto necesarias. Estaremos errados si subordinamos este debate a la lucha por el financiamiento de las investigaciones pero le daremos validez cuando discutamos cuál es la narrativa de la educación en nuestras universidades. Por otro lado, consideramos importante no excluir del debate el valor de la historia de la $\mathrm{HN}$ porque en su recorrido concebimos que la construcción del conocimiento científico es un proceso acumulativo que requiere imprescindiblemente de la experiencia previa, la cual puede resignificarse, tal como nos ha sucedido, como fuerza motivadora de la construcción del nuevo conocimiento.

Recorriendo las obras que construyeron el tejido del conocimiento en la HN, Alexander von Humboldt y Amado Bonpland representan la esencia del naturalista y de la HN. Estos dos grandes sabios expresan el punto exacto de equilibrio en el continuo debate científico que hemos planteado. Creemos que con su obra han simplificado parte de él en un hecho que en algún punto lo ha teñido de paradoja: demostraron que la descripción precisa y el vínculo emocional proporcionado por la experiencia del individuo son dos procesos intrínsecamente ligados para el desarrollo del conocimiento científico. Para nosotros, la filosofía científica de Humboldt y Bonpland representa el principal legado para la $\mathrm{HN}$, expresado así por Humboldt: "La percepción y la imaginación son tan necesarias como el pensamiento racional. Quienes quieran describir el mundo natural con la mera clasificación de las plantas, animales y rocas nunca lograrán acercarse a él”.

\section{Agradecimientos}

Este trabajo amplía un conjunto de ideas y reflexiones de nuestra disertación llevada a cabo en el Café de las Ciencias Humboldt \& Bonpland: el resplandor del conocimiento 
realizado el 29 de octubre de 2019 en el Museo de Ciencias Naturales Amado Bonpland de la Ciudad de Corrientes (Argentina), con motivo de la conmemoración del bicentenario del natalicio de Alexander von Humboldt. El Café de las Ciencias propició un espacio de júbilo en donde más de 150 concurrentes evocaron la memoria de Alexander von Humboldt y Amado Bonpland. Considérese este texto conmemorativo como un aporte figurativo que evidencia nuestra honra a sus legados. Nuestros agradecimientos al Dr. Frank Holl, por permitirnos tener el gran honor de compartir su sabiduría y calidez durante la conmemoración del natalicio de Humboldt en el Café Científico. A Eric Bourland (Agregado para la ciencia y la tecnología del Instituto Francés de Argentina de la Embajada de Francia) por su confianza para nuestra participación en el Café de las Ciencias. A la Cónsul Honoraria de Francia, Veronique Avart por su amable y cálido acompañamiento durante todo su trabajo de vinculación internacional. A Matthias Trager (Servicio Alemán de Intercambio Académico, DAAD) por su apoyo durante nuestra intervención en el Café de la Ciencia. A la Municipalidad de Corrientes y a su subsecretario de Ambiente, Alejandro Cristiá por su apoyo logístico y de vinculación interinstitucional. Al Instituto de Cultura de la Provincia de Corrientes y su presidente Arq. Gabriel Romero por el espacio del Museo de Ciencias Naturales Amado Bonpland brindado para el Café de la Ciencias. A Pedro Cuaranta por las ilustraciones de las figuras 1 y 2 . A los miembros de la Fundación Amado Bonpland y los amigos de la Asociación Cultural Bonpland con quienes compartimos la admiración por los dos sabios y su continuo aliento para este homenaje.

\section{Bibliografía}

ANDERSON, J. G. T. (2013). Deep things out of darkness. A History of Natural History. University of California Press, Berkeley, and Los Angeles \& London. https://doi.org/10.1525/california/9780520273764.001.0001

ANDERSON, J. G. T. (2017). Why ecology needs natural history. American Scientist 105: 290-297. https:// doi.org/10.1511/2017.105.5.290
ARNOLD, S. J. (2003). Too much natural history, or too little? Animal Behavior 65: 1065-1068. https://doi.org/10.1006/anbe.2003.2143

BARROWS, C. W., MURPHY-MARISCAL, M. L. \& HERNANDEZ, R. R. (2016). At a Crossroads: The Nature of Natural History in the Twenty-First Century. BioScience 66: 592-599. https://doi.org/10.1093/biosci/biw043

CAJADE, R., MEDINA, W., PIÑEIRO, J. M., COURTIS, A. C., ARGOITIA, M. A., CUARANTA, P., NUÑEZ, R. \& HERNANDO, A. (2019). Naturgemälde de los cerros Nazareno, Chico y Capará: síntesis del funcionamiento de los ecosistemas rocosos del Paraje Tres Cerros (Corrientes, Argentina). En ARBELO DE MAZZARO, A., G. D. MANGO DE RUBIO \& J. A. PERSI (eds.), II Congreso Internacional Interdisciplinario Aimé Bonpland-Alexander von Humboldt Año 2017, pp. 249-258. Instituto de Cultura, Moglia Ediciones, Corrientes.

DETTELBACH, M. (1999). The face of nature: Precise measurement, mapping, and sensibility in the work of Alexander von Humboldt. Studies in History Philosophy of Biological and Biomedical Sciences 30: 473-504.

https://doi.org/10.1016/S1369-8486(99)00011-4

FLEISCHNER, T. L. (2001). Natural history and the spiral of offering. 11 WILD EARTH, Fall/Winter 10-13.

FLEISCHNER, T. L. (2005). Natural history and the deep roots of resource management. Natural Resources Journal 45: 1-13. https://doi.org/10.1007/s11191-017-9880-8

FRANCIOLI, S. E. (2015). Historia natural: La discusión. Una revisión del concepto, el conflicto y sus ecos a la educación de las Ciencias Biológicas. Estudios Pedagógicos XLI, $\mathrm{N}^{\circ}$ Especial: 267-28.

https://doi.org/10.4067/S0718-07052015000300017

FUTUYMA, D. J. (1998). Wherefore and whither the Naturalist? The American Naturalist 151: 1-6. https://doi.org/10.1086/286097

GREENE, H. W. (2005). Organisms in nature as a central focus for biology. Trends in Ecology and Evolution 20: 23-27. https://doi.org/10.1016/j.tree.2004.11.005

HAYES, M. (2009). Into the field: naturalistic education and the future of conservation. Conservation Biology 23: $1075-1079$. https://doi.org/10.1111/j.1523-1739.2009.01302.x

HUMBOLDT, A. V. (1814-1829). Viaje a las regiones equinocciales del nuevo continente. Tomo III. Ediciones del Ministerio de Educación, Dirección de Cultura y Bellas Artes, Caracas, Venezuela. 
NOSS, R. F. (1996). The Naturalists are dying off. Conservation Biology 10: 1-3. https://doi.org/19.1046/j.1523-1739.1996.10010001.x PENCHASZADEH P. E. \& DE ASÚA, M. (2010). El deslumbramiento. Aimé Bonpland y Alexander von Humboldt en Sudamérica. Museo Argentino de Ciencias Naturales Bernardino Rivadavia, Buenos Aires, Argentina.

SCHMIDLY, D. J. (2005). What it means to be a naturalist and the future of natural history at American universities. Journal of Mammalogy 86: 449-456. https://doi.org/10.1644/1545-1542(2005)86[449:WI MTBA]2.0.CO;2
TEWKSBURY, J. J., JOHN, G. T., ANDERSON, J. G. T., BAKKER, J. D., BILLO, T. J., DUNWIDDIE, P. T., GROOM, M. J., HAMPTON, S. E., HERMAN, S. G., LEVEY, D. J., MACHNICKI, N. J., MARTÍNEZ DEL RIO, C., POWER, M. E., ROWELL, K. K., SALOMON, A. K., STACEY, L., TROMBULAK, S. C. \& WHEELE, T. A. (2014). Natural History's Place in Science and Society. BioScience 64: 300-310. https://doi.org/10.1093/biosci/biu032

WULF, A. (2016). La invención de la naturaleza. El nuevo mundo de Alexander von Humboldt. Taurus. Ciudad Autonoma de Buenos Aires, Argentina. 
